\title{
Widespread Cutaneous Metastasis as an Initial Clinical Presentation in Gastric Cancer
}

\author{
Sunigdha', Divya Khosla ${ }^{1 *}$, Kannan Periasamy ${ }^{1}$, Shruti Gupta ${ }^{2}$, Ojas Gupta ${ }^{2}$, Kirti Gupta ${ }^{2}$ and Rakesh Kapoor ${ }^{1}$ \\ ${ }^{1}$ Department of Radiotherapy, Postgraduate Institute of Medical Education and Research, Regional Cancer Centre, India \\ ${ }^{2}$ Department of Pathology, Postgraduate Institute of Medical Education and Research, India
}

Submission: June 08, 2020; Published: July 02, 2020

*Corresponding author: Dr Divya Khosla, Assistant Professor, Department of Radiotherapy, Postgraduate Institute of Medical Education and Research (PGIMER), Regional Cancer Centre, Chandigarh, India

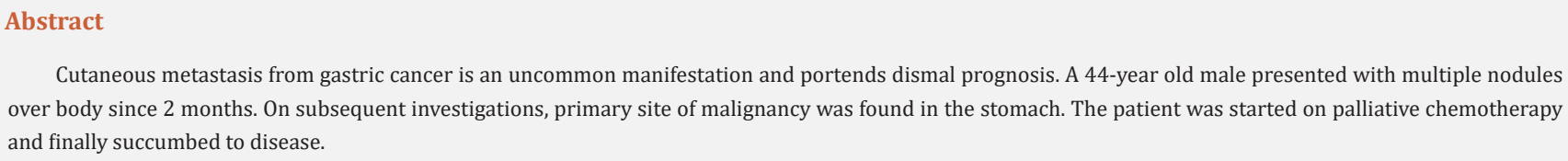

Cutaneous metastasis from gastric cancer is an uncommon manifestation and portends dismal prognosis. A 44-year old male presented with multiple nodules over body since 2 months. On subsequent investigations, primary site of malignancy was found in the stomach. The patient was started on palliative chemotherapy and finally succumbed to disease.

\section{Introduction}

Cutaneous metastases from gastrointestinal tract are rare and occur in the setting of advanced or disseminated disease. The most common primary site of origin of cutaneous metastasis is breast in females and lung in males [1]. Cutaneous metastases most commonly occur in the vicinity of the primary site of tumor [2]. Majority of these patients also have additional visceral metastasis at the time of presentation. These usually appear late in the course of disease but rarely may be the presenting sign. Gastric cancer is rarely reported as cause of widespread cutaneous metastasis at presentation. Herein, we report a rare case of widespread cutaneous metastasis as initial clinical presentation in gastric cancer.

\section{Case Presentation}

A 44-year-old male presented with complaints of multiple nodules over the body, abdominal discomfort, loss of appetite and loss of weight for 2 months. There was no history of difficulty in swallowing, abdominal pain, upper or lower gastrointestinal bleed or fever. Physical examination revealed multiple subcutaneous nodular lesions over neck, chest wall, abdominal wall, arms, back and buttocks (Figure 1a). On further evaluation, Contrast enhanced computed tomography (CECT) of the chest and abdomen showed multiple tiny nodules in bilateral lungs, multiple mediastinal lymph nodes, multiple soft tissue deposits in the anterior abdominal wall, left chest wall as well as intramuscular deposits in right gluteal muscle with suspicious mural thickening in gastroesophageal junction, cardia and body of stomach. PET-
CT revealed FDG avid thickening in the stomach with cutaneous nodules, skeletal and abdominal lymph nodes metastasis.

Upper gastrointestinal endoscopy showed an ulcerative mass at gastro-esophageal junction (GEJ) extending along lesser curvature and body of stomach. Endoscopic biopsy from the lesion was performed which was suggestive of adenocarcinoma of stomach (Figure 1b). Fine needle aspiration cytology of cutaneous lesion at neck was done which showed metastatic deposits (Figure 1c). Immunocytochemistry staining performed showed positivity for CK7 and CK 19 (Figure 1 d). The patient was explained about the disseminated disease in detail and was started on palliative chemotherapy with Docetaxel, cisplatin and 5FU after informed consent. The patient deteriorated after 3 cycles and died of progressive disease.

\section{Discussion}

The most common primary sites of origin of cutaneous metastasis are breast, lung, colon, oral cavity, kidney, and ovary [1]. Cutaneous metastasis from malignancies of upper gastrointestinal tract is extremely rare and reported to be less than 1\% [2]. Gastric cancer most commonly metastasizes to liver, lymph nodes and peritoneal cavity. Umbilical nodule (Sister Mary Joseph's nodule) is the most common cutaneous presentation from gastric cancer. When cutaneous involvement is present, most common affected sites are the chest and abdomen. Cutaneous metastasis to the extremities and multiple sites is uncommon. Metastatic dissemination to skin may occur through 


\section{Cancer Therapy \& Oncology International Journal}

the lymphatic channels or hematogenous spread. Our patient had multiple lesions involving the neck, chest, abdomen, back and thigh which indicates hematogenous spread of disease and is a rare manifestation of disease.

Cutaneous metastases usually appear late in the course of the disease but may also rarely be the presenting sign $[3,4]$ as in index case. The presence of cutaneous metastasis indicates disseminated disease and poor prognosis [2]. The intent of treatment in such cases is palliation and aimed at providing symptomatic relief and maintaining the quality of life. The present case emphasizes the importance of obtaining a detailed clinical history and keeping high index of suspicion for underlying gastric malignancy in a patient presenting with skin lesions and with history of loss of appetite and weight.

\section{Figure 1:}

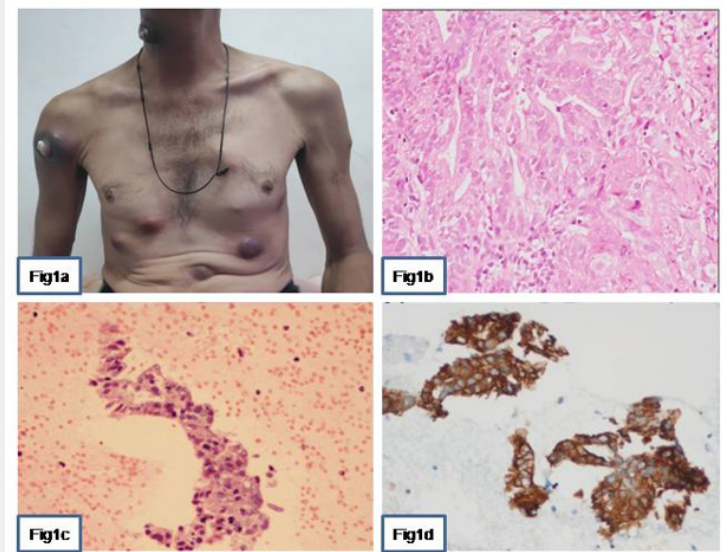

a. Multiple subcutaneous nodular lesions over neck, chest wall, abdominal wall, arms.

b. High magnification depicting tumor cells with vesicular chromatin and conspicuous nucleoli (H\&E x400).

c. Cell block sections showing tumor cells having moderately pleomorphic nuclei and moderate amount of cytoplasm.

d. Tumor cells showing strong cytoplasmic and membranous immunohistochemical staining for CK19.

\section{Conclusion}

Cutaneous metastasis in gastric cancer is a rare occurrence. It is a sign of advanced and disseminated disease. The prognosis is grave and treatment is aimed at achieving palliation. The present case is unique as the patient presented with widespread cutaneous metastasis at presentation with symptoms of loss of appetite and weight which prompted us to investigate for gastric malignancy.

\section{Conflicts of Interest Statement}

The author declares no conflicts of interest in the preparation of the manuscript.

\section{References}

1. Hu SC, Chen GS, Lu YW, Wu CS, Lan CC (2008) Cutaneous metastases from different internal malignancies: a clinical and prognostic appraisal. J Eur Acad Dermatol Venereol 22: 735-740.

2. Lookingbill DP, Spangler N, Helm KF (1993) Cutaneous metastases in patients with metastatic carcinoma: a retrospective study of 4020 patients. J Am Acad Dermatol 29: 228-236.

3. Xavier MH, Vergueiro Tde R, Vilar EG, Pinto JM, Issa MC, et al. (2008) Cutaneous metastasis of gastric adenocarcinoma: an exuberant and unusual clinical presentation. Dermatol Online J 14(11): 8.

4. Frey L, Vetter-Kauczok C, Gesierich A, Bröcker EB, Ugurel S (2009) Cutaneous metastases as the first clinical sign of metastatic gastric carcinoma. J Dtsch Dermatol Ges 7: 893-895.

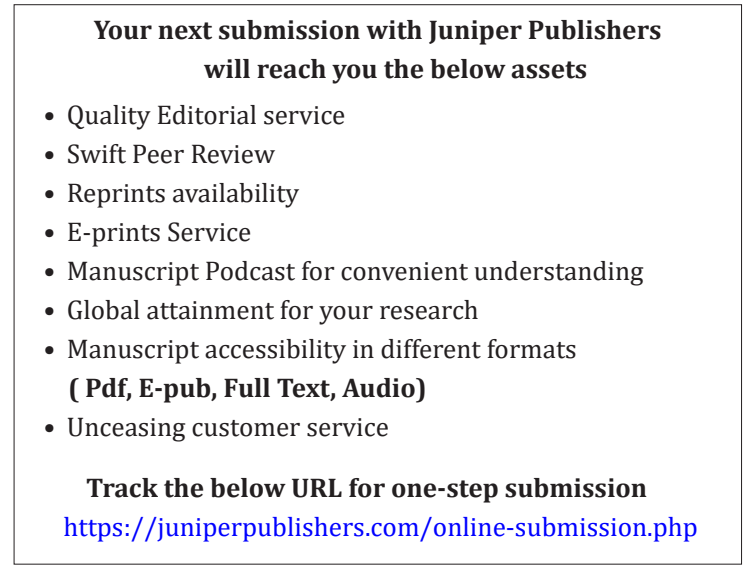

